\title{
Um estudo comparativo na geração de personas de crianças autistas com base em dados reais
}

\author{
Francisco Luciano Q. da Silva, Anna Beatriz Marques, Tatiane F. Figueiredo \\ Universidade Federal do Ceará (Campus Russas) - Russas, CE - Brasil \\ lucianoquirinol4@gmail.com, beatriz.marques@ufc.br, \\ tatianefernandesclia.ufc.br
}

\begin{abstract}
Studies have been proposing the automatic generation of personas, however, without exploring possible differences in the quality of the generated personas in relation to manual methods.This article reports a comparative study between the semiautomatic and manual generation of personas in relation to the quality of the personas obtained, using real data from autistic children. The obtained personas were evaluated for completeness, usability, clarity, and empathy. The results indicate that the semiautomatically generated personas showed similar quality to manually generated personas, but at a lower cost. This is expected to provide evidence of the potential benefits of this technique for software development.
\end{abstract}

Resumo. Estudos vêm propondo a geração de personas de forma automática, porém, sem explorar possiveis diferenças na qualidade das personas geradas em relação a métodos manuais. Este artigo relata um estudo comparativo entre a geração semiautomática e manual de personas em relação à qualidade das personas obtidas, utilizando dados reais de crianças autistas. As personas obtidas foram avaliadas em relação à completude, disposição de uso, clareza $e$ empatia. Os resultados indicam que as personas geradas de forma semiautomática apresentaram qualidade similar às personas geradas manualmente, porém a um custo menor. Com isso, espera-se fornecer indícios dos potenciais beneficios desta técnica para o desenvolvimento de software.

\section{Introdução}

A busca por métodos alternativos que auxiliem no tratamento do Transtorno do Espectro Autista (TEA) tem viabilizado o desenvolvimento de tecnologias que contribuem para amenizar as dificuldades enfrentadas por essas pessoas [Sousa et al. 2019]. O TEA é um distúrbio do neurodesenvolvimento e as suas características envolvem sintomas que afetam a socialização, comunicação e comportamento [Schmidt 2014]. Uma solução tecnológica eficiente para o TEA, requer conhecimento sobre significados, reações, críticas e comentários dos potenciais usuários [Braz et al. 2014].

A técnica de personas é uma relevante ferramenta para descobertas de informações sobre o usuário, por utilizar processos sucessivos de investigação sobre os usuários alvos [Leme et al. 2015]. Assim, a técnica de personas torna-se relevante para criação de tecnologias de apoio à intervenção sobre o TEA. De acordo com Jung et al. (2018) os métodos tradicionais de criação de personas representam um trabalho desafiador, por possuírem processos lentos e caros. Isso se dá, pelo fato de envolver estudos que produzem grande quantidade de informações para serem analisadas. 
Tentando solucionar este problema com o auxílio da Computação, surgiram os métodos de geração semiautomática e automática de personas.

Porém, os estudos que propõem a automatização da criação de personas não investigam a qualidade das personas geradas em comparação às personas geradas de forma tradicional e os custos dos processos. Deste modo, este estudo visa: (i) relatar a geração manual e semiautomática de personas de crianças autistas, (ii) descrever os insumos necessários para os processos e (iii) comparar a qualidade das personas criadas, segundo os critérios de completude, disposição de uso, clareza e empatia definidos por Salminen et al. (2020). Para a geração das personas foram utilizados dados reais de crianças autistas, obtidos de um survey conduzido por Sousa et al. (2019). Assim, esta pesquisa contribui para preencher esta lacuna sobre estudos relacionados à qualidade de personas, possibilitando também obter uma compreensão sobre o TEA.

\section{Fundamentação Teórica}

Segundo Masiero et al. (2012) personas são modelos criados para representar grupos de usuários, que permitem estabelecer um consenso entre projetistas e stakeholders, por possibilitarem um conhecimento sobre as motivações e necessidades dos usuários-alvo, podendo ser utilizada em qualquer fase do ciclo de vida de produtos interativos.

Uma metodologia amplamente utilizada para a criação de personas é o processo definido por Cooper et al. (2007), que busca identificar padrões de informações dos usuários em pesquisas. Para isso, é feita a organização dos dados, análise, seleção, identificação e sintetização de padrões, criação, análise e classificação das personas em diferentes tipos (Primária, Secundária, Suplementar, Cliente, Servida e Negativa).

A geração automática de personas é um método que envolve processos semi-automatizados ou automatizados, para a coleta e/ou análise de dados sobre os usuários utilizando Mineração de Dados [Filgueiras et al. 2005, Masiero et al. 2012, Leme et al. 2015]. Segundo Fayyad et al. (1996), Mineração de Dados é o processo de explorar uma base de dados em busca de padrões válidos, novos, úteis e compreensíveis para o proprietário da coleção. Como uma persona representa um grupo de usuários com características em comum, a Mineração de Dados pode apoiar no processo de identificação das similaridades contidas em uma base de dados, viabilizando a identificação de padrões para a criação de personas. A Clusterização é um método de Mineração de Dados adotado no processo de criação de personas automáticas, com o objetivo de particionar um conjunto de dados em grupos (clusters), de forma que os grupos sejam formados por dados similares entre si [Camilo e Silva 2009].

\section{Trabalhos Relacionados}

Masiero et al. (2012) adotaram a Clusterização em Python para criação automática de personas. O processo apresentado compreende: coleta de dados por meio de questionários; normalização dos dados; aplicação de algoritmo de Clusterização em Python; e combinação dos valores das variáveis. A técnica permite a análise de um grande volume de informações, que na geração manual poderiam ser mal aproveitadas.

Leme et al. (2015) também utilizou a Clusterização para gerar personas de forma automática. $\mathrm{O}$ estudo utiliza dados resultantes de survey aplicados com grupos de usuários da terceira idade do Facebook. A partir dos dados obtidos foi aplicada Clusterização, utilizando o algoritmo k-means, através da ferramenta WEKA, para 
identificar relações existentes entre os dados com o objetivo de identificar e definir personas. As personas identificadas foram avaliadas por meio de Design Participativo.

Jung et al. (2018) apresentam uma metodologia e sistema para geração automática de personas com base em dados de mídias sociais. A ferramenta permite a coleta, processamento e decomposição dos dados, produzindo variáveis descritivas para criar personas ricas de informações. A ferramenta possibilita a criação de personas a partir de uma larga escala de dados e permite a sua atualização com facilidade.

Em relação à personas sobre usuários autistas, Marques e Aires (2020) relatam o uso de netnografia para delinear personas de forma manual. Os autores utilizam a rede social Instagram para levantar dados de pessoas relacionadas ao TEA, por meio de observação e aplicação de questionários. A partir dos dados obtidos, personas relacionadas ao TEA (mãe e pai de autista, cuidadora, criança autista) foram criadas e utilizadas para elicitar requisitos. Os benefícios do processo adotado estão relacionados aos custos e alcance do público alvo para obtenção de informações.

Os trabalhos apresentados exploram abordagens de geração automática e manual, porém não comparam os resultados das técnicas. Apenas Leme et al. (2015) investiga a qualidade das personas geradas. O diferencial desta pesquisa é apresentar um estudo comparativo entre técnicas de geração manual e semiautomática de personas sobre a qualidade das personas geradas.

\section{Criação de Personas}

A metodologia utilizada para a criação das personas foi criada com base em Cooper et al. (2007), com adaptações para a criação semiautomática e são apresentadas nesta seção com os resultados obtidos ao longo das etapas. Para a criação das personas, utilizou-se uma base de dados resultante de um survey sobre o uso de tecnologias de software por pessoas autistas, conduzido no estudo de Sousa et al. (2019).

\subsection{Ajustes do Material}

Esta etapa visa preparar os dados do survey de Sousa et al. (2019) para a criação de personas nesta pesquisa. As atividades foram baseadas em Cooper et al. (2007).

Seleção de variáveis significativas: foi realizada uma análise do survey com o objetivo de selecionar variáveis significativas para compor a persona. Com isto, a base de dados com as respostas do survey foi adaptada para manter somente os dados referentes às variáveis selecionadas. Como os resultados do survey continham somente informações sobre crianças autistas, foi observado que só seria possível criar personas do tipo primária, que são personas que representam o alvo principal para o projeto.

Definição da estrutura da persona: com base nas variáveis selecionadas foi criado um template de persona com campos e definições (Figura 1). O template foi usado para orientar a criação de personas e possibilitar a comparação dos resultados.

Mapeamento das variáveis: as variáveis significativas selecionadas do survey foram mapeadas com os campos do template definido, especificando quais informações deveriam compor cada campo das personas (Figura 1). 


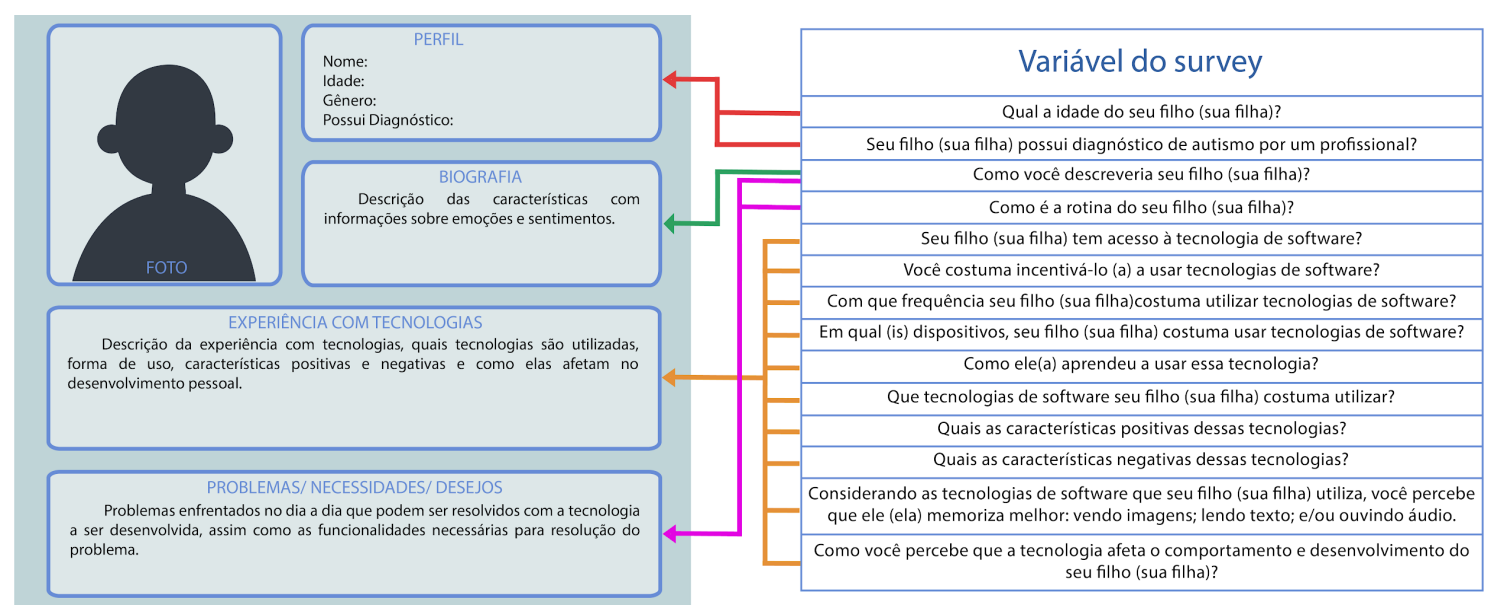

Figura 1. Mapeamento das variáveis com o template das personas.

\subsection{Seleção e Treinamento}

A seleção e treinamento de participantes para a criação manual de personas foi necessária para reduzir o viés do pesquisador, que realizou os ajustes no material.

Seleção dos participantes: Para a criação manual de personas, foram selecionados três estudantes de um curso de graduação em Engenharia de Software com experiência em projetos acadêmicos que envolvem criação de persona e pesquisas sobre TEA. Por sua vez, o método de criação semiautomática foi conduzido pelo primeiro autor deste artigo. Durante a adoção do método semiautomático, o primeiro autor não teve acesso às personas resultantes do método manual, apenas identificou a quantidade de personas criadas, para gerar a mesma quantidade de personas semiautomáticas para efeitos de comparação dos resultados.

Treinamento dos participantes: os participantes selecionados para adotar o método de criação manual receberam treinamento sobre o processo de Cooper et al. (2007) que foi adotado nesta pesquisa. Para a criação semiautomática não foi necessário treinamento devido ao processo ser conduzido por um dos pesquisadores.

\subsection{Criação Manual}

O método utilizado para identificação e elaboração das personas de forma manual foi adaptado de Cooper et al. (2007) e todas as atividades deste método foram realizadas pelos participantes selecionados, sem a intervenção dos pesquisadores.

Identificação de padrões de comportamentos significativos: foi realizada a análise da base de dados buscando identificar grupos de conteúdos que se apresentavam em vários intervalos dentro das variáveis. Para isto, inicialmente foi considerada a variável idade, posteriormente foi levado em consideração as variáveis de questões fechadas, identificando os padrões. Este procedimento foi realizado em 2 horas.

Sintetização das características e objetivos relevantes: foi realizada uma análise dos padrões identificados na etapa anterior e uma filtragem dos mesmos considerando as variáveis de questões abertas, para identificar semelhanças existentes. Posteriormente foram selecionados os padrões mais significativos, definindo a quantidade de personas que seriam necessárias. Com base nas informações de cada padrão selecionado foi feito o preenchimento do template definido para as personas. 
Durante o processo, foram criadas seis personas, nomeadas pelo grupo responsável pelo processo como: Vinícius, Adrian, Ícaro, Mariana, Yasmin e Maria Clara. Este processo levou 1 hora e 50 minutos para ser realizado.

Verificação da integridade e redundância: as personas criadas foram analisadas para identificar a necessidade de complementar ou remover informações. Uma persona (Maria Clara) foi removida e as outras cinco personas (Vinícius, Adrian, Ícaro, Mariana e Yasmin) foram atualizadas. Este procedimento foi realizado em 30 minutos. Todas as personas estão disponíveis em relatório técnico ${ }^{1}$.

$\mathrm{Na}$ Figura 2 é apresentada a persona Adrian, uma criança autista de 6 anos, calma com pouca concentração que frequenta a escola e terapias. Tem experiência com tecnologia e seus pais o incentivam a utilizar. Utiliza Smartphone para jogar e assistir vídeos no YouTube. O uso de tecnologias possibilitou o desenvolvimento da fala e melhorou a concentração, porém o tempo de uso é um fator preocupante para os pais. Adrian possui problemas de fala, coordenação motora e concentração.

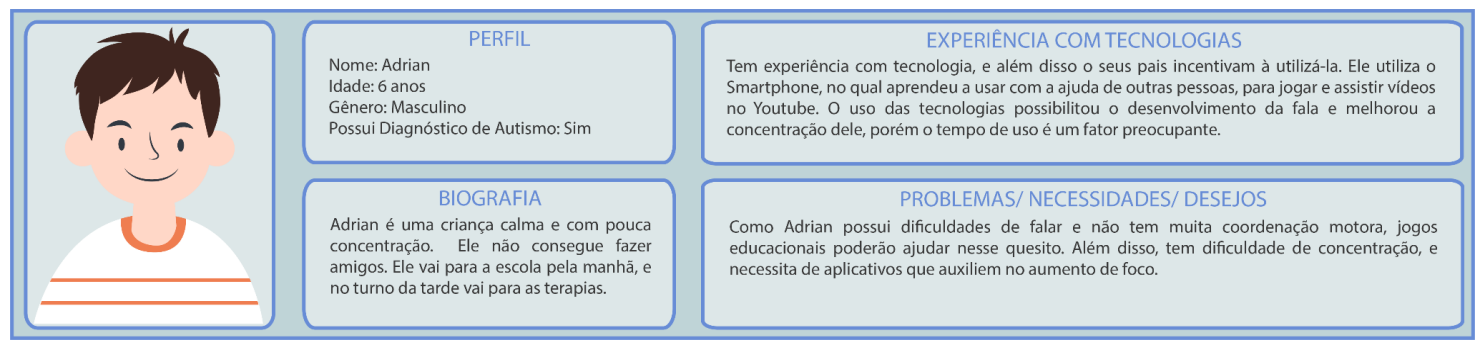

Figura 2. Persona Adrian.

\subsection{Criação Semiautomática}

A criação semiautomática de personas foi baseada em Clusterização conforme proposto por Masiero et al. (2012) e Leme et al. (2015) para a criação de personas. Todas as atividades desta etapa foram realizadas pelo primeiro autor do artigo.

Preparação dos Dados: foi realizado o pré-processamento da base de dados, buscando a padronização de termos sinônimos para garantir eficácia na Clusterização. Este procedimento foi executado em 1 hora e 30 minutos.

Formação de clusters: para a formação de clusters e identificação de padrões existentes na base de dados foi utilizada a ferramenta WEKA $^{2}$ com o algoritmo $k$-means, que utiliza o conceito de centróide e busca os diferentes centros existentes no conjunto de dados. Cada centróide representa um cluster. O valor $k$ é recebido como parâmetro do algoritmo e é utilizado como base para calcular pontos que representam os "centros" [Camilo e Silva 2009]. Para uma correta execução do algoritmo k-means foi necessário a definição do parâmetro $k$, que recebeu o valor 5 , com base no número de personas geradas no processo de criação manual, para fins de comparação entre os métodos. Então, a base de dados foi inserida na ferramenta WEKA, que executou a clusterização em 0,06 segundos. Os cinco clusters gerados pela ferramenta WEKA estão disponíveis em relatório técnico ${ }^{3}$.

\footnotetext{
${ }^{1}$ bit.ly/3tfw $7 \mathrm{hf}$

${ }^{2}$ www.cms.waikato.ac.nz/ml/weka/

${ }^{3}$ bit.ly/3rPmllO
} 
Criação das personas: as informações contidas nos clusters identificados na etapa anterior foram mapeadas e adicionadas ao template definido para as personas. Neste processo foram formadas cinco personas, nomeadas de Yuri (Cluster 0), Melissa (Cluster 1), Luan (Cluster 2), Laura (Cluster 3) e Davi (Cluster 4). Este procedimento levou 30 minutos para ser executado.

Enriquecimento das personas: as informações das personas foram analisadas para identificar a necessidade de complementação. Com base na interpretação das informações dos clusters as informações foram complementadas. Este procedimento foi executado em 1 hora. Todas as personas estão disponíveis em relatório técnico ${ }^{4}$.

A Figura 3 apresenta a persona Yuri, uma criança autista de 4 anos, hiperativa e determinada, que gosta de desafios, descobrir coisas novas e quer realizar suas atividades sozinho. Possui acesso à tecnologia e costuma receber incentivos para utilização. Através de experimentação aprendeu sozinho a utilizar smartphone e usa frequentemente para acessar aplicativos. As tecnologias têm ajudado Yuri no aprendizado e desenvolvimento de habilidades, porém um dos pontos negativos que tem sido observado pelos pais é o vício causado. Yuri aprende bastante vendo imagens. A tecnologia pode ajudar no controle das emoções de Yuri, assim como no seu desenvolvimento, sendo uma alternativa para complementação de estudos e terapias.

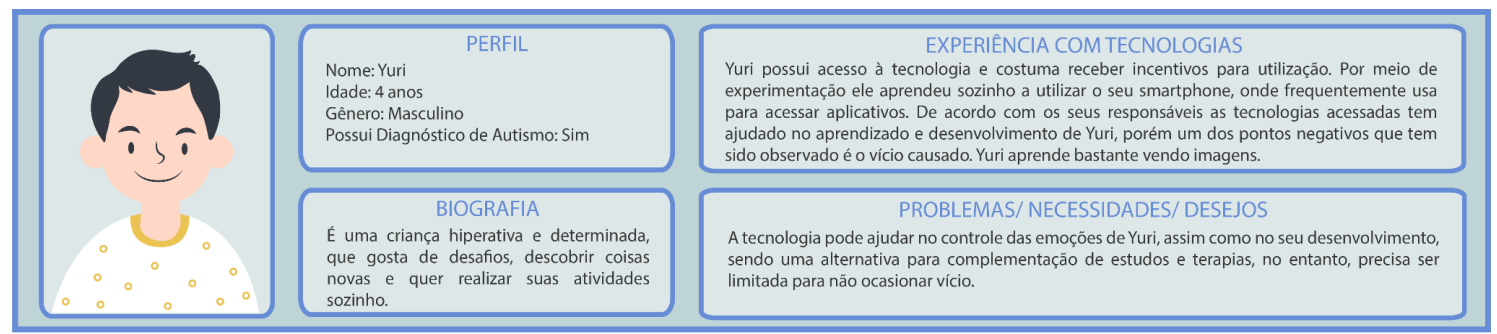

Figura 3. Persona Yuri.

\section{Avaliação da qualidade das personas}

Esta pesquisa teve caráter exploratório e visou responder a seguinte questão de pesquisa: Qual a qualidade de personas criadas por meio do método semiautomático em comparação com o método manual? Com o intuito de avaliar a qualidade das personas criadas por meio de cada método, foi conduzida uma avaliação com profissionais relacionados ao TEA. O instrumento de avaliação foi adaptado do questionário para avaliação de personas proposto por Salminen et al. (2020). As etapas seguidas neste processo de avaliação e os resultados obtidos são descritos a seguir:

Seleção de critérios para avaliação das personas: os critérios e questões para avaliação de personas definidos por Salminen et al. (2020) foram analisados para selecionar os critérios aplicáveis a esta pesquisa. Os critérios selecionados foram completude (CO) - medir quão bem a persona apresenta informações essenciais sobre os usuários que descreve; disposição de uso (DU) - medir quão disposto o entrevistado está para utilizar a persona; clareza (CL) - medir quão claramente a informação da persona é apresentada; empatia (EM) - medir quão bem o entrevistado se familiariza com a persona. A Tabela 1 apresenta as questões associadas aos critérios selecionados, conforme proposto por Salminen et al. (2020).

\footnotetext{
${ }^{4}$ bit.ly/3viHEhy
} 
Tabela 1. Questões selecionadas para avaliação das personas.

\begin{tabular}{|l|}
\hline \multicolumn{1}{|c|}{ Questão } \\
\hline CO1 - O perfil da persona é detalhado o suficiente para tomar decisões sobre as personas que ele descreve. \\
\hline CO2 - O perfil da persona parece completo. \\
\hline CO3 - O perfil da persona fornece informações suficientes para entender as pessoas que ela descreve. \\
\hline CO4 - No perfil da persona não faltam informações essenciais. \\
\hline DU1 - Eu usaria essa persona em pesquisas e práticas profissionais que envolvem pessoas com TEA. \\
\hline $\begin{array}{l}\text { DU2 - Posso imaginar maneiras de usar as informações da persona em pesquisas e práticas profissionais que envolvem pessoas } \\
\text { com TEA. }\end{array}$ \\
\hline DU3 - Essa persona melhoraria minha capacidade de tomar decisões sobre as pessoas que ela descreve. \\
\hline CL1 - As informações sobre a persona são bem apresentadas. \\
\hline CL2 - O texto no perfil da persona é claro o suficiente para ser lido. \\
\hline CL3 - As informações no perfil da persona são fáceis de entender. \\
\hline EM1 - Sinto que entendo essa persona. \\
\hline EM2 - Sinto fortes laços com essa persona. \\
\hline EM3 - Eu posso imaginar um dia na vida dessa persona. \\
\hline
\end{tabular}

Criação de instrumento de avaliação: a partir dos critérios e questões selecionadas foram criados dois formulários no Google Formulários para avaliação das personas. Como foram obtidas dez personas na pesquisa, decidiu-se dividi-las em dois grupos de cinco personas e distribuí-las aos avaliadores. Dois formulários foram criados com as mesmas questões, porém com personas diferentes. No formulário A existiam três personas obtidas pelo processo manual e duas personas obtidas pelo processo semiautomático: Adrian, Ícaro, Mariana, Melissa e Davi. O formulário B continha três personas obtidas do processo semiautomático e duas personas obtidas pelo processo manual: Yuri, Luan, Laura, Vinícius e Yasmin. Para a avaliação das questões do formulário foi utilizada uma escala de concordância de cinco pontos: discordo totalmente; discordo; indiferente; concordo; e concordo totalmente.

Seleção dos avaliadores: A avaliação foi realizada por um grupo de dez profissionais relacionados ao TEA, com atuação profissional na saúde (4), educação (2) ou pesquisa (4). As experiências destes profissionais com TEA variam de menos de 1 ano até mais de 5 anos trabalhando com o assunto. Os profissionais da área de pesquisa possuem experiência em estudos relacionados à IHC.

Condução da avaliação: Os avaliadores foram organizados em dois grupos: (i) grupo A, que receberam o formulário A; e (ii) grupo B, que receberam o formulário B. A distribuição dos grupos aconteceu aleatoriamente, mas mantendo o balanceamento dos grupos em relação às áreas de atuação (Tabela 2). Os formulários de avaliação foram enviados para os avaliadores por e-mail, descrevendo o objetivo da avaliação, a definição de termos (personas e TEA) e prazos para conclusão da avaliação. Cada avaliador analisou cinco personas.

Análise dos resultados da avaliação: Os dados obtidos foram tabulados e analisados quantitativamente. Valores foram atribuídos para a escala de concordância utilizada no instrumento de avaliação, da seguinte forma: discordo totalmente $=1$, discordo $=2$, indiferente $=3$, concordo $=4 \mathrm{e}$ concordo totalmente $=5$. Esta transformação possibilitou medir a qualidade, de forma que quanto maior fosse a pontuação maior seria a qualidade geral da persona. 
Tabela 2. Distribuição dos avaliadores.

\begin{tabular}{|c|c|c|c|c|c|}
\hline \multicolumn{3}{|c|}{ Grupo A } & \multicolumn{3}{|c|}{ Grupo B } \\
\hline ID & Perfil & Personas validadas & ID & Perfil & Personas validadas \\
\hline A1 & Pesquisa & \multirow{5}{*}{$\begin{array}{l}\text { Adrian, Ícaro, Mariana, } \\
\text { Melissa e Davi. }\end{array}$} & A6 & Pesquisa & \multirow{5}{*}{$\begin{array}{c}\text { Yuri, Luan, Laura, Vinícius e } \\
\text { Yasmin. }\end{array}$} \\
\hline A2 & Pesquisa & & A7 & Pesquisa & \\
\hline $\mathrm{A} 3$ & Saúde & & A8 & Educação & \\
\hline A4 & Educação & & A9 & $\overline{\text { Saúde }}$ & \\
\hline A5 & Saúde & & A10 & Saúde & \\
\hline
\end{tabular}

Seguindo as recomendações de Salminen et al. (2020) para medir a qualidade das personas, os valores relacionados à escala de concordância foram divididos heuristicamente em faixas, da seguinte maneira: qualidade baixa $(1<=$ qualidade $<=2)$; qualidade média $(2<$ qualidade $<4)$; qualidade alta $(4<=$ qualidade $<=5)$.

Para obtenção do indicador de qualidade por critério, a média das respostas para as questões de cada critério foi calculada e, em seguida, uma média por critério foi obtida (colunas CO, DU, CL e EM da Tabela 3). Para obter um indicador da qualidade geral de cada persona, foi calculada a média das notas dos critérios de qualidade (coluna Qualidade geral da Tabela 3). A Tabela 3 apresenta um resumo dos indicadores de qualidade obtidos para as personas.

Tabela 3. Resumo dos indicadores de qualidade das personas.

\begin{tabular}{|c|c|c|c|c|c|c|c|}
\hline Método & Persona & CO & DU & CL & EM & Qualidade geral & Qualidade da persona \\
\hline \multirow{5}{*}{ Semiautomático } & Laura & 3,30 & 3,87 & 3,60 & 3,93 & 3,68 & Qualidade média \\
\cline { 2 - 8 } & Davi & 2,40 & 2,27 & 3,60 & 2,93 & 3,05 & Qualidade média \\
\cline { 2 - 8 } & Luan & 3,60 & 3,93 & 4,13 & 3,60 & 3,82 & Qualidade média \\
\cline { 2 - 8 } & Melissa & 2,05 & 2,73 & 3,00 & 2,67 & 2,61 & Qualidade média \\
\cline { 2 - 8 } & Yuri & 3,10 & 3,60 & 4,20 & 3,47 & 3,52 & Qualidade média \\
\hline \multirow{5}{*}{ Manual } & Vínicius & 2,25 & 2,80 & 3,87 & 3,27 & 3,05 & Qualidade média \\
\cline { 2 - 8 } & Adrian & 2,75 & 3,53 & 3,67 & 3,13 & 2,27 & Qualidade média \\
\cline { 2 - 8 } & Ícaro & 2,40 & 3,20 & 3,13 & 2,80 & 2,88 & Qualidade média \\
\cline { 2 - 7 } & Mariana & 2,60 & 3,53 & 3,60 & 3,07 & 3,20 & Qualidade média \\
\cline { 2 - 7 } & Yasmin & 2,45 & 3,40 & 3,73 & 3,27 & 3,21 & Qualidade média \\
\hline
\end{tabular}

Por outro lado, para obter um indicador da qualidade das personas por método (semiautomático e manual) foi calculada a média das notas obtidas pelas personas por critério, esses resultados são apresentados na Figura 4.

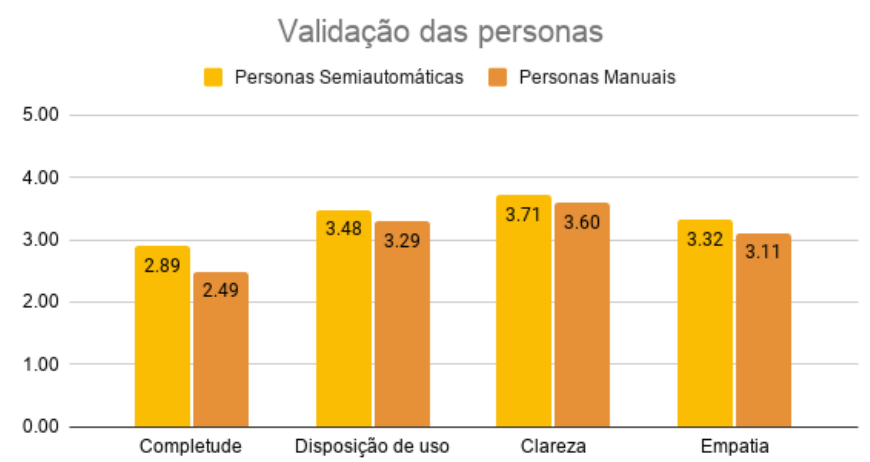

Figura 4. Resultado sobre a qualidade dos métodos por critérios. 
As personas manuais apresentaram valores de qualidade menores em relação às personas semiautomáticas para todos os critérios. Contudo, todas as personas foram consideradas de média qualidade para todos os critérios, o que indica que a diferença de valores não influenciou na classificação da qualidade das personas. Ao conduzir o teste de hipóteses t-test, apresentado em relatório técnico ${ }^{5}$, não obteve-se diferença significativa nos critérios de qualidade de personas manuais e semiautomáticas.

Em relação à completude, que analisa o quão bem a persona apresenta as informações essenciais sobre os usuários que descreve, o valor indica que o conteúdo das personas poderia ser mais explorado. Sobre a disposição de uso, foi analisado quão dispostos os avaliadores estariam a utilizar às personas. De acordo com observações feitas pelos avaliadores A3 e A9, do perfil da saúde, as informações apresentadas pelas personas são incompletas e alguns termos utilizados são inadequados para a área, o que pode influenciar na disposição de uso das personas. Para o critério de clareza obteve-se melhores resultados, foi analisado quão claramente as informações da persona estavam sendo apresentadas. Em relação à empatia, buscou-se medir quão bem os avaliadores se familiarizaram com as personas. Este critério está relacionado à forma com que as personas transmitem as informações e são percebidas. A falta de informações e a utilização de termos inadequados, relatadas pelos profissionais da área da saúde podem ter impedido a obtenção de melhores resultados para os métodos no critério empatia.

Os resultados indicam que o método semiautomático é uma abordagem viável, principalmente quando se considera questões como volume de dados a serem analisados, tempo, equipe envolvida e custo. Para a execução do método manual foram empregadas 4 horas e 20 minutos por cada participante, totalizando 13 horas (4h20min x 3 participantes). Para a execução do método semiautomático foram empregadas no total de 3 horas e 0,06 segundos. Os participantes do processo de criação manual das personas relataram que a experiência foi cansativa devido à quantidade de dados a serem analisados e durante o processo de identificação dos padrões, surgiram dúvidas relacionadas à escolha de parâmetros ideais. Tais problemas podem ser minimizados com o uso do método semiautomático apresentado nesta pesquisa.

\section{Conclusões}

Este artigo apresentou um estudo comparativo na geração de personas semiautomáticas e manuais em relação a critérios de qualidade. Para isto foi utilizada uma base de dados com características de crianças autistas, conduzido por Sousa et al. (2019). No método de geração manual foi adotado uma adaptação de Cooper et al. (2007) e o processo de geração foi conduzido por um grupo de três estudantes de graduação em Engenharia de Software com experiência em TEA e IHC. O método de geração semiautomática adotou uma adaptação de Cooper et al. (2007) com a metodologia de Clusterização, ferramenta WEKA e algoritmo $k$-means, sendo o processo conduzido pelo primeiro autor do artigo.

Para realizar a análise comparativa da qualidade das personas, uma avaliação das personas geradas foi conduzida com a participação de dez profissionais relacionados ao TEA, com atuação profissional na saúde, educação ou pesquisas em IHC. Como resposta à questão de pesquisa, os resultados mostraram uma similaridade entre a qualidade das personas geradas nos métodos semiautomático e manual. Todas as personas criadas obtiveram qualidade média para todos os critérios avaliados. O método

\footnotetext{
${ }^{5}$ bityli.com/yOdLS
} 
semiautomático apresentou valores ligeiramente superiores para todos os critérios em relação ao método manual. $\mathrm{O}$ teste de hipóteses t-test indicou que essa diferença não é significativa. Apesar dos métodos apresentarem resultados similares em relação à qualidade das personas, questões como quantidade de pessoas envolvidas, custo e tempo necessário para adoção do método devem ser consideradas. Estes fatores indicam que a adoção de métodos semiautomáticos pode trazer benefícios para as pesquisas que adotam este tipo de metodologia para geração de personas.

\section{Referências}

Braz, P., Raposo, A., and de Souza, C. S. (2014). Uso de design probes no design de tecnologias para terapeutas de crianças com autismo. In Proc. of the 13th Brazilian Symposium on Human Factors in Computing Systems, pages 140-149. SBC.

Camilo, C. O. and Silva, J. C. d. (2009). Mineração de dados: Conceitos, tarefas, métodos e ferramentas. Universidade Federal de Goiás (UFG), pages 1-29.

Cooper, A., Reimann, R., and Cronin, D. (2007). About face 3: the essentials of interaction design. John Wiley \& Sons.

Fayyad, U., Piatetsky-Shapiro, G., and Smyth, P. (1996). The kdd process for extracting useful knowledge from volumes of data. Communications of the ACM, 39(11):27-34.

Filgueiras, L., Aquino Jr, P., Sakai, R., Filho, Á. G., Torres, C., and Barbarian, I. (2005). Personas como modelo de usuários de serviços de governo eletrônico. In Proc. of the 2005 Latin American conference on Human-computer interaction, pages 319-324.

Jung, S.-g., Salminen, J., Kwak, H., An, J., and Jansen, B. J. (2018). Automatic persona generation (apg): A rationale and demonstration. In Proc. of the 2018 Conference on Human Information Interaction \& Retrieval, pages 321-324. ACM.

Leme, R., Zaina, L., and Casadei, V. (2015). Um estudo da interação do público da terceira idade com o facebook em dispositivos móveis: identificação e definição das pessoas. In Proc. of the 16th Brazilian Symposium on Human Factors in Computing Systems IHC, volume 15, pages 53-62.

Marques, A. B. and Aires, G. (2020). Uso da netnografia para a geracão de personas e requisitos para sistemas com foco em pessoas com transtorno do espectro autista: Um relato de experiência. In Anais do V Workshop sobre Aspectos Sociais, Humanos e Econômicos de Software, pages 1-10. SBC.

Masiero, A. A., Ferreira, L. A., and Aquino Jr, P. T. (2012). Algoritmos de clusterização e python científico apoiando modelagem de usuário. In Companion Proc. of the 11th Brazilian Symposium on Human Factors in Computing Systems, pages 47-50. SBC.

Salminen, J., Santos, J. M., Kwak, H., An, J., Jung, S.-g., and Jansen, B. J. (2020). Persona perception scale: Development and exploratory validation of an instrument for evaluating individuals' perceptions of personas. International Journal of Human-Computer Studies, vol. 141, p. 102437.

Schmidt, C. (2014). Autismo, educação e transdisciplinaridade. Papirus Editora.

Sousa, T. A., Ferreira, V. D., and dos S. Marques, A. B. (2019). How do software technologies impact the daily of people with autism in brazil: A survey. In Proc. of the XV Brazilian Symposium on Information Systems, pages 1-8. 